\title{
Analysis of T-helper type 1 and 2 cells and T-cytotoxic type 1 and 2 cells of sentinel lymph nodes in breast cancer
}

\author{
KATSUHIKO EHI $^{1}$, SUMIYA ISHIGAMI ${ }^{1}$, IZUMI MASAMOTO ${ }^{2}$, YOSHIKAZU UENOSONO ${ }^{1}$, SHOJI NATSUGOE ${ }^{1}$, \\ TAKAAKI ARIGAMI $^{1}$, HIDEO ARIMA ${ }^{1}$, YUKO KIJIMA ${ }^{1}$, HEIJI YOSHINAKA ${ }^{1}$, SHIGEHIRO YANAGITA ${ }^{1}$ \\ TSUTOMU KOZONO $^{1}$, YAWARA FUNASAKO ${ }^{1}$, IKURO MARUYAMA ${ }^{2}$ and TAKASHI AIKOU ${ }^{1}$ \\ ${ }^{1}$ Digestive Surgery Surgical Oncology, Kagoshima University School of Medicine, 8-35-1 Sakuragaoka, \\ Kagoshima 890-8520; ${ }^{2}$ Department of Laboratory and Vascular Medicine, Kagoshima University \\ Graduate School of Medicine, 8-35-1 Sakuragaoka, Kagoshima-shi, Kagoshima 890-8544, Japan
}

Received August 10, 2007; Accepted October 1, 2007

\begin{abstract}
The immune suppression of sentinel lymph node (SN) is directly influenced by primary tumors. It has been reported that the T-helper type 1 (Th1) to T-helper type 2 (Th2) ratio or T-cytotoxic type 1 (Tc1) to T-cytotoxic type 2 (Tc2) ratio of lymph node lymphocytes could be used to evaluate direct immunological circumstances. We attempted to evaluate the Th1 to Th2 cell and Tc1 to Tc2 cell balance in $\mathrm{SN}$ and non-SN and to clarify the immunological status of sentinel nodes in breast cancer. To evaluate this balance, $\mathrm{SN}$ and non-SN were identified by radioguided methods and lymph node lymphocytes were collected, and prepared for flow cytometry. The Th1 to Th2 and Tc1 to Tc 2 ratio were calculated by 3-color flow cytometry. The ratio of SN and non-SN was compared. The results demonstrated that $\mathrm{SN}$ was detected in 24 out of 24 patients $(100 \%)$. As regards the correlation between $\mathrm{SN}$ and non-SN in the same patients, the Th1 to Th2 ratio in SN was significantly lower than that in non-SN for all patients $(\mathrm{p}<0.05)$. Among clinicopathological factors, a larger tumor diameter, histology and nodal involvement affected the decreased Th1 to Th2 ratio in SN significantly $(\mathrm{p}<0.05)$. We reached the conclusion that the increasing immunosuppressive conditions derived from the tumor may deteriorate the Th1 to $\mathrm{Th} 2$ ratio of $\mathrm{SN}$ in an earlier stage as compared with non-SN. According to the tumor extension and nodal involvement, the Th1 to Th2 ratio in $\mathrm{SN}$ was significantly suppressed. The current result supports that mainly helper T-cell paralysis occurred first in SN and this seems to be a favorable condition in forming lymph node metastases in SN. Moreover, the immunologic interaction
\end{abstract}

Correspondence to: Dr Sumiya Ishigami, Digestive Surgery Surgical Oncology, Kagoshima University, 8-35-1 Sakuragaoka, Kagoshima 890-8520, Japan

E-mail: ishiga@m.kufm.kagoshima-u.ac.jp

Key words: T-helper type 1, T-helper type 2, sentinel node, breast cancer between the primary site and regional lymph nodes may serve as useful criteria for identifying sentinel nodes.

\section{Introduction}

Sentinel lymph node (SN) is one of the recent clinical topics in the surgical field. $\mathrm{SN}$ is defined as the first drainage lymph node from the primary cancer (1). The exploration of the metastatic status of SN enables us to perform individualized surgical management and a clinical application of SN navigation surgery was started for melanoma and breast cancer (2-4). It has been reported that the immune modulation of SN occurred in breast cancer (5), melanoma (6) and gastric cancer (7). These results suggested that immunological paralysis may occur first in $\mathrm{SN}$ as compared with non-SN. However, technically it seems difficult to directly prove the immunosuppressive circumstance in $\mathrm{SN}$.

T-helper type 1 (Th1) and 2 (Th2) and T-cytotoxic type 1 (Tc1) and 2 cells (Tc2) play important immunoregulatory roles in cancer patients $(8,9)$. Th1 and Tc1 cells activate a cellular immunological response through increased interferon (IFN) $-\gamma$ or interleukin (IL)-2 production. Th2 and Tc2 cells suppress cellular immunity through increased IL-4 and IL-10 production. It was reported that the Th1 to Th2 or Tc1 to Tc2 ratio of the lymphocytes in peripheral blood reflected an immunosuppressive condition directly in cancer patients. As a result of the investigation of the Th1 to Th2 ratio in tumorinfiltrating lymphocytes in lung cancer, the Th1 to Th2 ratio decreased, according to the tumor progression (10). However, there have been a few reports which disclose the Th1 to Th2 ratio of SN lymphocytes with respect to the intracellular cytokine level using flow cytometry. In the present study, we investigated the Th1 to Th2 and Tc1 to Tc2 ratio in connection with $\mathrm{SN}$ and clinical factors.

\section{Materials and methods}

Patients. A total of 24 patients with primary breast cancer who underwent curative operation with lymph node dissection, including sentinel node exploration in Kagoshima University Hospital were enrolled in the current study. All of the patients 
were evaluated to be free from nodal involvement before surgery. Clinicopathological features were evaluated according to the TNM classification of breast tumors (11).

Seven patients received partial mastectomy and 10 patients had total mastectomy. The numbers of patients with less than Level I, Level I and II lymphadenectomy were 5, 10 and 9, respectively. Final pathology revealed that 5 patients had lymph node metastasis. Five and 3 patients were positive for estrogen and progesterone receptor, respectively. A histological examination revealed that 3 patients had non-invasive cancer and the remaining 21 had invasive carcinomas (Table I).

Exploration and detection of sentinel lymph nodes. The sentinel nodes were detected by a radioguided method as previously reported (12). In brief, $2 \mathrm{ml}$ of Tc-labeled tin colloid was injected into the peritumoral sites of the subdermal space $12 \mathrm{~h}$ before surgery. During surgery, the radioisotope uptake in each lymph node was measured using Navigator GPS (Tyco Healthcare, Ltd, Tokyo, Japan). The radioisotope uptake in all dissected lymph nodes was measured again after surgery. When counts for individual lymph nodes were 10 times greater than background levels, the lymph node was identified as an SN. Sentinel nodes were identified in all patients (detection rate: $100 \%$ ). After lymph node dissection, between $\mathrm{SN}$ and non-SN in the same lymph node station number, half of them were used for pathological examination and the remainder were used for flow cytometric analysis.

Cell separation and stimulation. A set of lymphocytes of SN and non-SN from each patient was collected and minced in a culture medium that consisted of RPMI-1640 (Gibco, Grand Island, NY) supplemented with $10 \%$ fetal bovine serum (FBS), $50 \mathrm{U} / \mathrm{ml}$ penicillin, and $50 \mathrm{~g} / \mathrm{ml}$ streptomycin. Prepared cells $\left(10^{7}\right)$ of lymph node lymphocytes were incubated for $2 \mathrm{~h}$ at $37^{\circ} \mathrm{C}$ in a flat culture bottle. Adherent cells were removed with mild centrifugation. Then, they were used for regional lymph node lymphocytes.

Lymph node lymphocytes were stimulated with phorbol 12-myristate 13 -acetate (PMA) $25 \mathrm{ng} / \mathrm{ml}$ plus ionomycin $1 \mathrm{~mol} / \mathrm{l}$ in the presence of brefeldin A $10 \mathrm{~g} / \mathrm{ml}$ (Sigma) in 24-well plates at a concentration of $2 \times 10^{6}$ cells $/ \mathrm{ml}$ for $5 \mathrm{~h}$ at $37^{\circ} \mathrm{C}$ with $5 \% \mathrm{CO}_{2}$ in RPMI-1640 supplemented with FBS.

Antibodies. The determination of antibodies, procedure of intracellular staining and flow cytometric analysis were followed according to a previous report (10). Phycoerythrin cyanine 5 (PE-Cy5)-conjugated anti-CD4 and PE-Cy5conjugated anti-CD8, PE-Cy5-conjugated anti-mouse immunoglobulin (Ig) G1, fluorescein isothiocyanate (FITC)conjugated anti-IFN- and phycoerythrin (PE)-conjugated anti-IL-4, unlabeled anti-IFN and unlabeled anti-IL-4 were obtained from Becton-Dickinson Immunocytometry Systems (BDIS, San Jose, CA) and used to analyze cell surface antigens and intracellular cytokines.

Intracellular cytokine staining. The stimulating cells were washed in phosphate-buffered saline (PBS) with $0.5 \%$ bovine serum albumin (BSA). Anti-CD4-PECy5 or anti-CD8PECy5 antibody was added to the lymphocytes and incubated for $20 \mathrm{~min}$ at $4^{\circ} \mathrm{C}$ and the cells were then washed
Table I. Patient background.

\begin{tabular}{lc}
\hline Clinical factors & Patient number \\
\hline Age & $38-76$ (average 57.4 yrs) \\
Gender & \\
Female & 24 \\
Male & 0
\end{tabular}

Tumor diameter

Tis 3

$\mathrm{Tl} \quad 13$

T2 6

T3 1

$\mathrm{T} 4$

Nodal involvement

Yes 6

No 18

Histology

Non-invasive 3

Invasive 21

Operation method

Mastectomy 11

Partial resection $\quad 13$

Degree of lymphadenectomy

SN biopsy 6

Level I 13

Level II 5

Number of sentinel nodes

1

$2-5$

$3 \quad 4$

$\geq 4 \quad 2$

with PBS plus $0.5 \%$ BSA. The cell pellet was fixed and permeabilized using a Fix and Perm kit (Dako, USA). AntiIFN- FITC and anti-IL-4 PE were added and incubated for 20 min at room temperature. As controls for the intracellular cytokine detection, mouse IgG1 FITC was added to the cell pellets and incubated for $20 \mathrm{~min}$ at $4{ }^{\circ} \mathrm{C}$. The cell pellets were washed with PBS plus $0.5 \%$ BSA and fixed and permeabilized with the above-mentioned Fix and Perm kit. Unlabeled antibodies (anti IFN- and anti IL-4 antibody, Pharmingen) were used for blocking special binding. IFNFITC and IL-4-PE were added without washing and incubated for $20 \mathrm{~min}$ at room temperature. Then, the cells were analyzed by flow cytometry.

Flow cytometry. Three-color flow cytometric analysis was performed on a FACSCalibur flow cytometer (BDIS). Data were acquired using CellQuest software (BDIS). Twenty thousand cells were analyzed for the cell surface marker and intracellular cytokine analysis. We defined the cell 
CD4

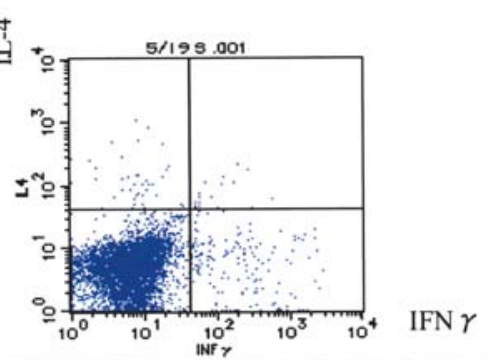

SN

non SN

苞

CD4

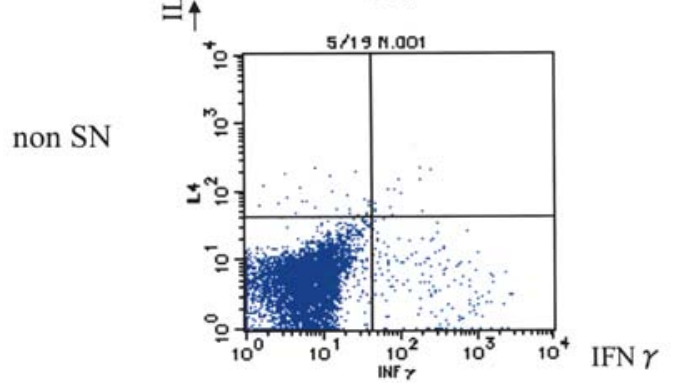

CD8

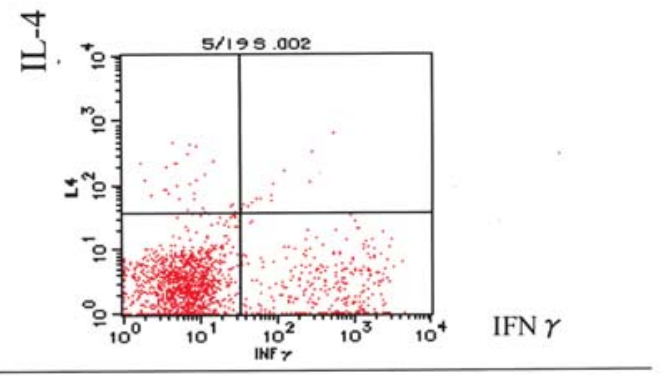

CD8

$\stackrel{=1}{+1}$

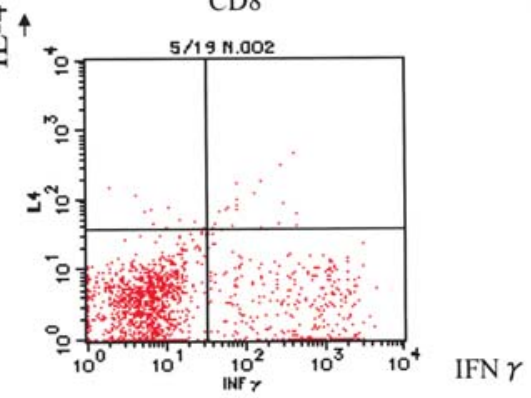

Figure 1. Three-color flow cytometric analysis of SN and non-SN for Th1 to Th2 and Tc1 to Tc2 balance.

1) Percentage of Th1 lymphocytes

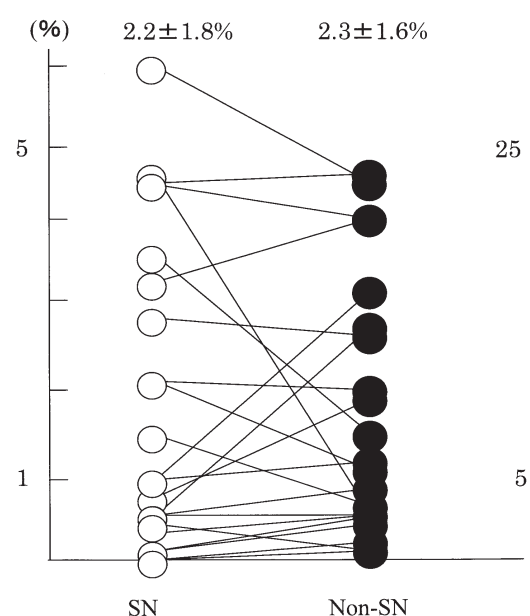

3) Percentage of Th2 lymphocytes

2) Percentage of Tc1 lymphocytes

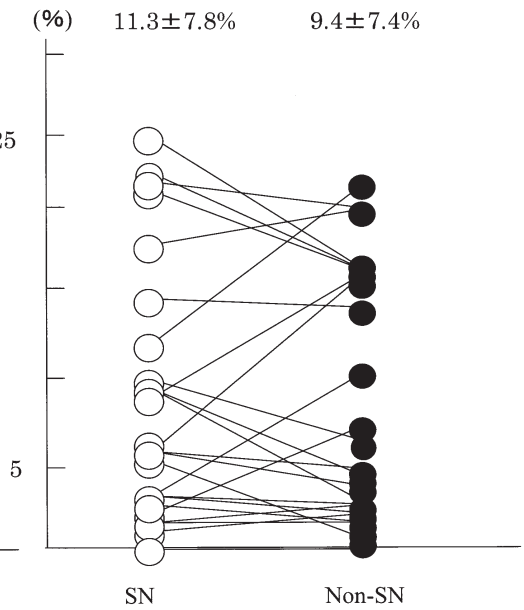

4) Percentage of Tc2 lymphocytes

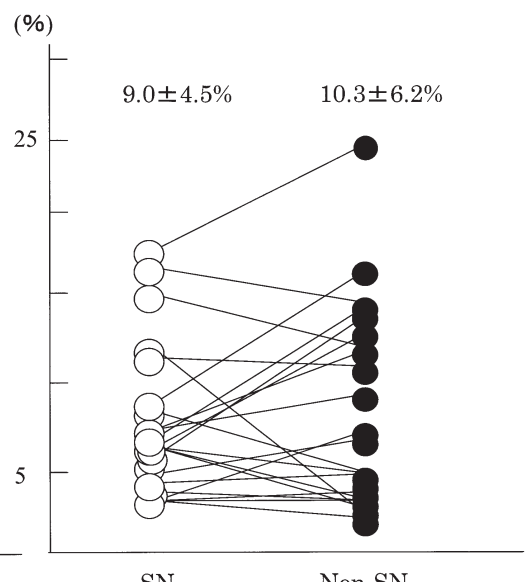

$(\%)$

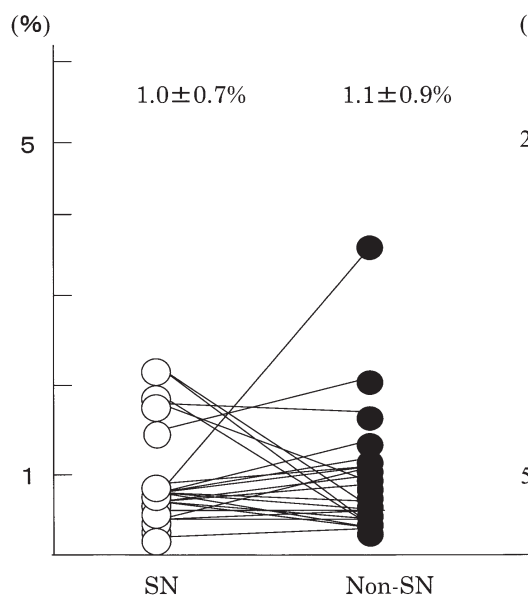

SN

Figure 2. Comparison of Th1 and Tc1 lymphocytes between SN and non-SN. 


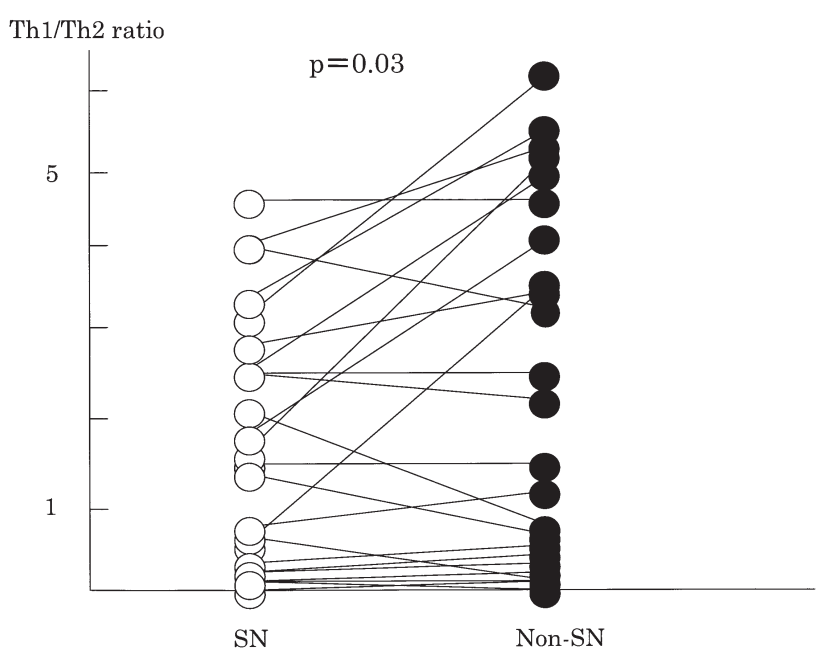

Figure 3. Comparison of the Th1 to Th2 ratio between SN and non-SN.

populations as follows: populations on $\mathrm{CD}^{+}$cells: Th1, IFNpositive and IL-4-negative; Th2, IFN-negative and IL-4positive. Populations on $\mathrm{CD} 8^{+}$cells were defined as: $\mathrm{Tc} 1$, IFN-positive and IL-4-negative; Tc2, IFN-negative and IL-4positive.

Statistical analysis. Differences among the study groups were assessed using an unpaired and paired Student's t-test because the variables had parametric meaning. Statistical significance was defined as $\mathrm{p}<0.05$.

\section{Results}

SN status. The number of SNs ranged from 1 to 4 (average 2.4). In 6 out of $24(25 \%)$ SNs, nodal involvement was found by intraoperative histological examination. According to the histological result during surgery, lymph node dissection was converted from sentinel biopsy to level I lymphadenectomy in 2 patients.

Th1, Th2, Tc1 and Tc2 lymphocytes in SN and non-SN. According to flow cytometric analysis, Th1, Th2, Tc1 and Tc2 lymphocytes were defined and the percentage was calculated (Fig. 1). The percentage of Th1, Th2, Tc1 and Tc2 lymphocytes in $\mathrm{SN}$ and non-SN was calculated. Average percentages of Th1, Tc1, Th2 and Tc2 lymphocytes in SN were $2.2 \pm 1.8,11.3 \pm 7.8,1.0 \pm 0.7$ and $9.0 \pm 4.5$, respectively and those in non-SN were $2.3 \pm 1.6,9.4 \pm 7.4,1.1 \pm 0.9$ and $10.3 \pm 6.2$, respectively. The Th1 and Tc1 lymphocytes in $\mathrm{SN}$ tended to be higher than that in non-SN. In contrast, the Th2 and Tc2 lymphocytes in SN tended to be lower than in the non-SN. However, there was no statistical significance (Fig. 2). When comparing the Th1 to Th2 ratio between $\mathrm{SN}$ and non-SN, it was significantly lower in $\mathrm{SN}$ than in non-SN (p=0.03) (Fig. 3). Confined to the case with the tumor diameter $\geq 20 \mathrm{~mm}$ and invasive carcinoma, the Th1 to Th2 ratio of SN was significantly lower in SN than in non-SN ( $\mathrm{p}=0.02)$ (Fig. 4). The presence or absence of nodal involvement did not affect the Th1 to Th2 ratio (Fig. 5). In contrast, there was no significant difference of the Tc1 to Tc2 ratio between SN and non-SN (Fig. 6). The Tc1 to $\mathrm{Tc} 2$ ratio was not affected by nodal involvement and tumor diameter (data not shown).

The Th1 to Th2 and Tc1 to Tc2 ratio in SN or non-SN and clinicopathological factors. The Th1 to Th2 and Tc1 to Tc2 ratio in $\mathrm{SN}$ and non-SN were analyzed by 4 clinical factors (age, tumor diameter, nodal involvement and tumor histology). In SNs, a significantly high Th1 to Th2 ratio was found in patients with age $<50$, tumor size $<1.5 \mathrm{~cm}$ or invasive carcinoma ( $\mathrm{p}<0.05, \mathrm{p}<0.05$ and $\mathrm{p}<0.01$, respectively). In

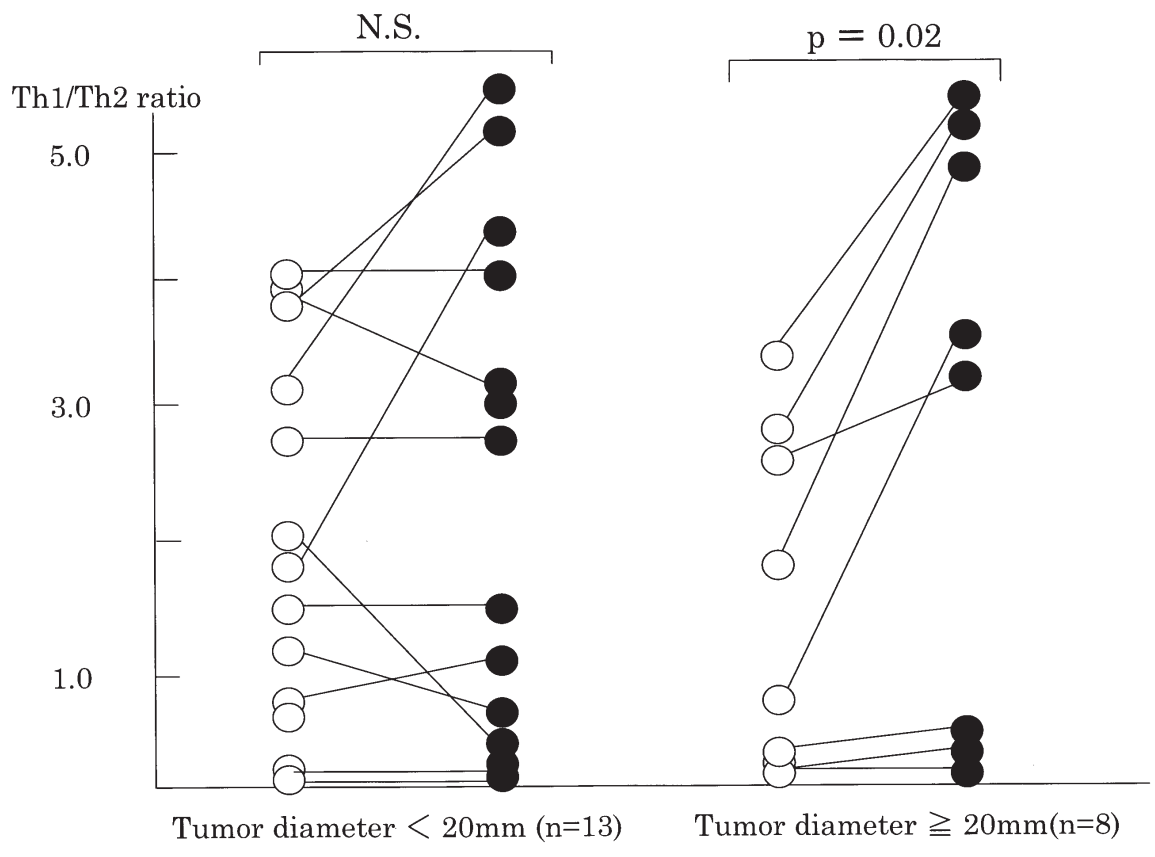

Figure 4. Comparison of the Th1 to Th2 ratio between SN and non-SN divided by tumor diameter. 


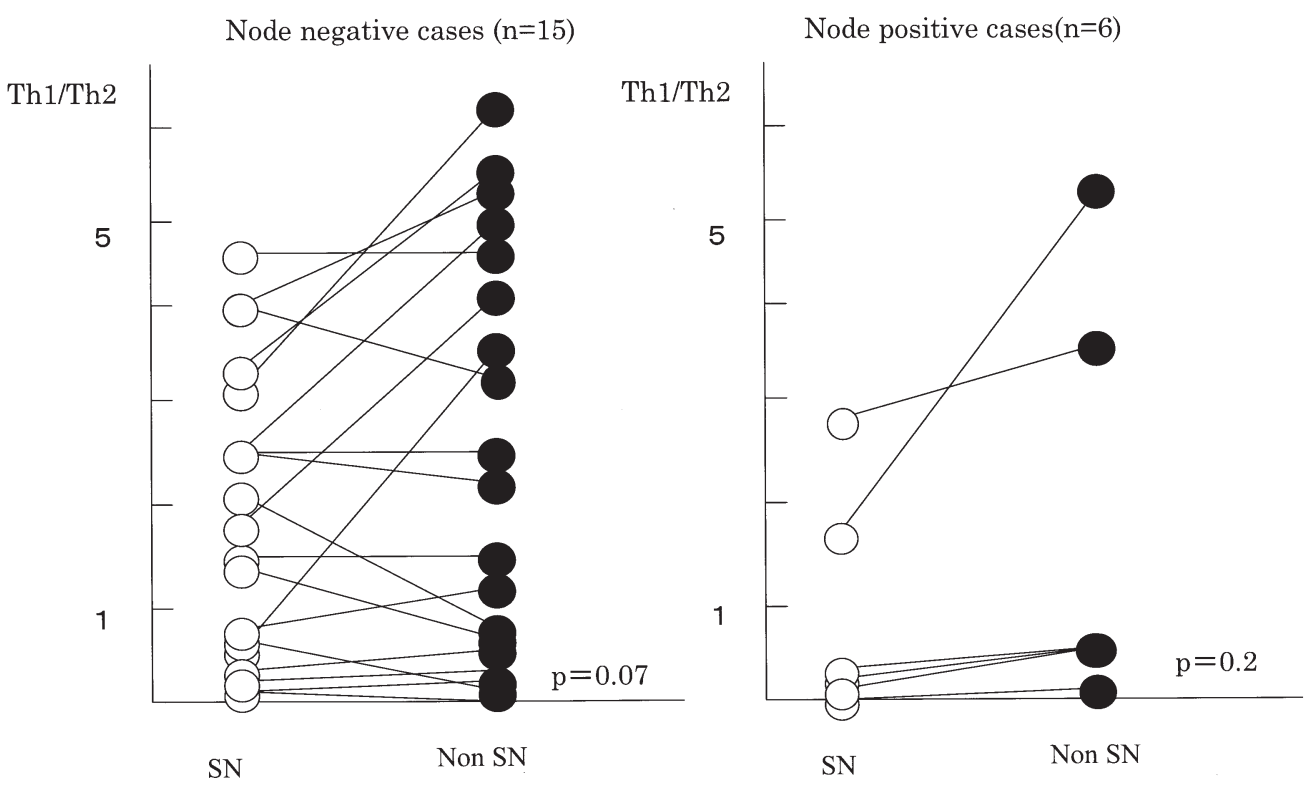

Figure 5. Comparison of the Th1 to Th2 ratio between SN and non-SN divided by nodal involvement.

Table II. Th1 to Th2 and $\mathrm{Tcl}$ to $\mathrm{Tc} 2$ ratio in $\mathrm{SN}$ according to clinicopathological factors.

\begin{tabular}{|c|c|c|c|c|c|}
\hline Clinical factors & & Thl/Th2 ratio & $\mathrm{p}$ value & $\mathrm{Tcl} / \mathrm{Tc} 2$ & $\mathrm{p}$-value \\
\hline \multicolumn{6}{|l|}{ Age } \\
\hline$<50$ & $\mathrm{n}=10$ & $1.8 \pm 1.3$ & \multirow[t]{2}{*}{$\mathrm{p}<0.05$} & $9.2 \pm 8.5$ & \multirow[t]{2}{*}{ n.s. } \\
\hline$\geq 50$ & $\mathrm{n}=14$ & $1.1 \pm 1.0$ & & $8.1 \pm 8.0$ & \\
\hline \multicolumn{6}{|l|}{ Tumor diameter ${ }^{\mathrm{a}}$} \\
\hline$<1.5 \mathrm{~cm}$ & $\mathrm{n}=9$ & $2.8 \pm 1.2$ & \multirow[t]{2}{*}{$\mathrm{p}<0.05$} & $9.8 \pm 8.4$ & \multirow[t]{2}{*}{ n.s. } \\
\hline$\geq 1.5 \mathrm{~cm}$ & $\mathrm{n}=12$ & $1.4 \pm 1.1$ & & $9.3 \pm 9.2$ & \\
\hline \multicolumn{6}{|c|}{ Nodal involvement ${ }^{\mathrm{a}}$} \\
\hline Yes & $\mathrm{n}=6$ & $0.8 \pm 1.3$ & \multirow[t]{2}{*}{$\mathrm{p}<0.05$} & $5.6 \pm 5.6$ & \multirow[t]{2}{*}{ n.s. } \\
\hline No & $\mathrm{n}=15$ & $2.3 \pm 1.3$ & & $10.1 \pm 9.5$ & \\
\hline \multicolumn{6}{|l|}{ Histology } \\
\hline Non-invasive & $n=3$ & $0.35 \pm 0.28$ & \multirow[t]{2}{*}{$\mathrm{p}<0.01$} & $3.5 \pm 1.1$ & \multirow[t]{2}{*}{$\mathrm{p}<0.01$} \\
\hline Invasive & $\mathrm{n}=21$ & $1.9 \pm 1.4$ & & $8.8 \pm 8.5$ & \\
\hline
\end{tabular}

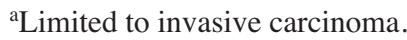

patients without nodal metastasis, the Th1 to Th2 ratio of SN was also significantly higher compared with patients with nodal metastasis $(\mathrm{p}<0.05)$ (Table II). However, in the non-SN, a significant difference was not found in age, tumor size and lymph node metastasis (Table III). The Th1 to Th2 and Tc1 to Tc2 ratio was significantly higher in invasive carcinoma than in non-invasive carcinoma $(\mathrm{p}<0.01)$.

\section{Discussion}

The Th1 to Th2 ratio, which was thought to reflect the immunological function of helper T-cells, was significantly lower in SNs than in non-SNs. This result suggested that immunological paralysis occurred in SN. Cochran et al also reported that the number of dendritic cells decreased in $\mathrm{SN}$ in melanoma (6). When immune competent cells were analyzed by RT-PCR and flow cytometry using markers such as HL-DR, CD80, CD83 and IFN- $\gamma$, the level of infiltration of such cells significantly decreased in SN compared with non-SNs in breast cancer (13). In the current study, the significant differences between SN and non-SN were prominent in patients with invasive breast cancer tumors $\geq 20 \mathrm{~mm}$ in size. This result was in agreement with the previous report that the immunological paralysis of helper T-cells occurred according to tumor proliferation.

The patients with a large tumor size showed a low Th1 to $\mathrm{Th} 2$ ratio in SN. It was suggested that as the tumor grew, immunosuppressive substances from the tumor were secreted 
Table III. Th1 to Th2 and Tcl to Tc2 ratio in non-SN according to clinicopathological factors.

\begin{tabular}{|c|c|c|c|c|c|}
\hline Clinical factors & & Th1/Th2 ratio & $\mathrm{p}$ value & $\mathrm{Tcl} / \mathrm{Tc} 2$ & p-value \\
\hline \multicolumn{6}{|l|}{ Age } \\
\hline$<50$ & $\mathrm{n}=10$ & $2.4 \pm 1.9$ & \multirow[t]{2}{*}{ n.s. } & $9.1 \pm 9.6$ & \multirow[t]{2}{*}{ n.s. } \\
\hline$\geq 50$ & $\mathrm{n}=14$ & $1.5 \pm 1.3$ & & $8.6 \pm 9.2$ & \\
\hline \multicolumn{6}{|l|}{ Tumor diameter ${ }^{\mathrm{a}}$} \\
\hline$<1.5 \mathrm{~cm}$ & $\mathrm{n}=9$ & $3.2 \pm 1.8$ & \multirow[t]{2}{*}{ n.s. } & $8.5 \pm 6.8$ & \multirow[t]{2}{*}{ n.s. } \\
\hline$\geq 1.5 \mathrm{~cm}$ & $\mathrm{n}=12$ & $2.6 \pm 2.1$ & & $11.6 \pm 11.8$ & \\
\hline \multicolumn{6}{|c|}{ Nodal involvement ${ }^{\mathrm{a}}$} \\
\hline Yes & $\mathrm{n}=6$ & $1.6 \pm 2.1$ & \multirow[t]{2}{*}{ n.s. } & $5.7 \pm 7.6$ & \multirow[t]{2}{*}{ n.s. } \\
\hline No & $\mathrm{n}=15$ & $3.0 \pm 1.9$ & & $10 \pm 11.0$ & \\
\hline \multicolumn{6}{|l|}{ Histology } \\
\hline Non-invasive & $\mathrm{n}=3$ & $0.3 \pm 0.1$ & \multirow[t]{2}{*}{$\mathrm{p}<0.01$} & $3.9 \pm 4.2$ & \multirow[t]{2}{*}{$\mathrm{p}<0.01$} \\
\hline Invasive & $\mathrm{n}=21$ & $2.6 \pm 2.0$ & & $9.3 \pm 9.5$ & \\
\hline
\end{tabular}

${ }^{\text {a }}$ Limited to invasive carcinoma.

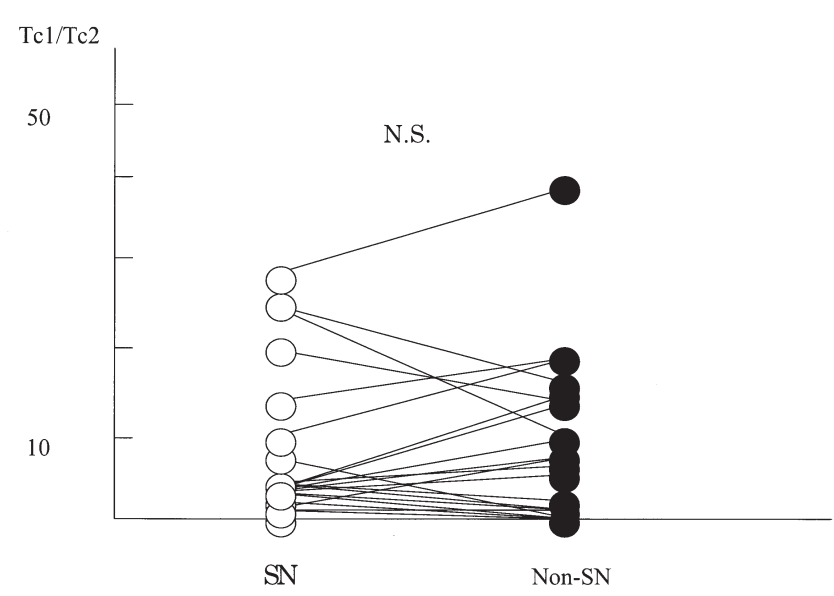

Figure 6. Comparison of the Tc1 to Tc2 ratio between $\mathrm{SN}$ and non-SN.

and drained to the SN. The Th1 to Th2 ratio in $\mathrm{SN}$ or non-SN may be influenced by the tumor volume. Ito et al reported that the Th1 to Th2 ratio in tumor-infiltrating lymphocytes (TILs), but not in peripheral blood, correlated with clinical factors in lung cancer (10). In this context, an immunosuppressive circumstance may first occur in locally affected places such as the SN or TIL, but not in the non-SN or peripheral blood lymphocytes. As for lymph node metastases in SN, Matsuura et al analyzed the 58 breast cancer patients. They concluded that the T-cell immunological status in SN was up-regulated after metastasis was overt, although it was depressed before the metastasis (13). In the current study, the $\mathrm{Th} 1$ to $\mathrm{Th} 2$ ratio of $\mathrm{SN}$ in node-positive patients was significantly lower than that in node-negative patients. Since node-positive SN lymphocytes coexisted with tumor cells, it was speculated that tumor cells directly suppress the immune system.
Contrary to the Th1 to Th2 ratio, no significant difference was found in the Tc1 to Tc2 ratio between $\mathrm{SN}$ and non-SN and among the clinical factors. Ito et al reported that the Tc1 to $\mathrm{Tc} 2$ ratio in not only lymph node but also peripheral blood lymphocytes did not affect clinical variables such as the tumor diameter and nodal involvement in lung cancer patients, which was similar to our results (10). The difference of the Tc1 to Tc2 ratio for tumor proliferation and lymph node metastasis may suggest that the Tc1 to Tc2 ratio is stable in any clinical stage. Immunological paralysis may initially start from the helper T-cell subset imbalance.

In the current study, we had 3 non-invasive carcinoma patients who showed a significantly lower Th1 to Th2 ratio of $\mathrm{SN}$, as compared with invasive carcinoma patients. Considering the tumor biological behavior, these results did not unexpectedly reflect the tumor aggressiveness of the noninvasive carcinoma. However, the tumor volume of these non-invasive carcinoma in 3 patients was larger than that of the remaining 21 patients with invasive carcinomas and thus, a significantly lower level of the Th1 to Th2 ratio may depend on the tumor volume.

Schule et al showed that a significant loss of the CD3- $\zeta$ chain expression of T cells in SN occurred at the early tumor stage in breast cancer (14). In the current study, the population density of Tc1-positive cells was preserved in $\mathrm{SN}$; however, cytotoxic $\mathrm{T}$ cells did not function well because of impaired CD3 complex molecules. It has been reported that celecoxib up-regulates the expression of the $\zeta$ chain of the T-cell receptor (15). The normalization of not only the T-cell subset imbalance, but also the $\mathrm{CD} 3-\zeta$ chain expression may be essential to retrieving intranodal anti-tumor immunoreaction, which prevents or destroys nodal involvement.

In conclusion, our data suggested that the helper T-cell deterioration was augmented by tumor proliferation and lymph node metastases. We clarified that the functional change of the helper T-cell immunity first occurred in SN lymphocytes in 
breast cancer. Accordingly, SNs were directly influenced by immunosuppressive molecules from the tumor and SNs were in preferential condition for nodal involvement. The tumorinduced immune modulation of SLNs facilitates lymph node metastases by deteriorating helper $\mathrm{T}$ balance in breast cancer. The immune modulation of the lymph nodes may also serve as useful criteria for identifying sentinel nodes (16).

\section{References}

1. Morton DL, Wen DR, Wong JH, Economou JS, Cagle LA, Storm FK, Foshag LJ and Cochran AJ: Technical details of intraoperative lymphatic mapping for early stage melanoma. Arch Surg 127: 392-399, 1992.

2. Krag DN, Meijer SJ, Weaver DL, Loggie BW, Harlow SP, Tanabe KK, Laughlin EH and Alex JC: Minimal-access surgery for the staging of malignant melanoma. Arch Surg 130: 654-658,1995.

3. Uren RF, Thompson JF and Howman-Giles R: Sentinel-lymph node biopsy in breast cancer. Lancet 352: 1472-1473, 1998.

4. Giuliano AE, Haigh PI, Brennan MB, Hansen NM, Kelley MC, Ye W, Glass EC and Turner RR: Prospective observational study of sentinel lymphadenectomy without further auxillary dissection in patients with sentinel node-negative breast cancer. J Clin Oncol 18: 2553-2559, 2000.

5. Huang RR, Wen DR, Guo J, Giuliano AE, Nguyen M, Offodile R, Stern S, Turner R and Cochran AJ: Selective modulation of paracortical dendritic cells and T-lymphocytes in breast cancer sentinel lymph nodes. Breast J 6: 225-232, 2000.

6. Cochran AJ, Morton DL, Stern S, Lana AM, Essner R and Wen DR: Sentinel lymph nodes show profound down-regulation of antigen-presenting cells of the paracortex: implications for tumor biology and treatment. Mod Pathol 14: 604-608, 2001.

7. Ishigami S, Natsugoe S, Uenosono Y, Hata Y, Nakajo A, Miyazono F, Matsumoto M, Hokita S and Aikou T: Infiltration of antitumor immunocytes into the sentinel node in gastric cancer. J Gastrointest Surg 7: 735-739, 2003.
8. Gazzinelli RT, Makino M, Chattopadhyay SK, Snapper CM, Sher A, Hugin AW and Morse HC III: CD4 ${ }^{+}$subset regulation in viral infection. Preferential activation of Th2 cells during the progression of retrovirus-induced immunodeficiency in mice. J Immunol 148: 182-188, 1992.

9. Belardelli F: Role of interferons and other cytokines in the regulation of the immune response. APMIS 103: 161-179, 1995.

10. Ito N, Nakamura H, Tanaka Y and Ohgi S: Lung carcinoma: analysis of T-helper type 1 and 2 cells and T-cytotoxic type 1 and 2 cells by intracellular cytokine detection with flow cytometery. Cancer 85: 2359-2367, 1999.

11. Sobin LH and Wittekind C (eds): TNM Classification of Malignant Tumours. 5th edition Wiley-Liss, New York, 1997.

12. Kijima Y, Yoshinaka H, Natsugoe S, Ehi K, Arigami T and Uenosono S: Surgical treatment for solitary recurrence of breast cancer to the mammary lymph nodes. Med J Kagoshima Univ 57: 19-25, 2005

13. Matsuura K, Yamaguchi Y, Ueno H, Osaki A, Arihiro K and Toge T: Maturation of dendritic cells and T-cell responses in sentinel lymph nodes from patients with breast carcinoma. Cancer 106: 1227-1236, 2006.

14. Schule J, Bergkvist L, Hakansson L, Gustafsson B and Hakansson A: Down-regulation of the CD3-zeta-chain in sentinel node biopsies from breast cancer patients. Breast Cancer Res Treat 74: 33-40, 2002.

15. Ferrandina G, Ranelletti FO, Legge F, Salutari V, Martinelli E, Fattorossi A, Lorusso D, Zannoni G, Vellone V, Paglia A and Scambia G: Celecoxib up-regulates the expression of the zetachain of T-cell receptor complex in tumor-infiltrating lymphocytes in human cervical cancer. Clin Cancer Res 12: 2055-2060, 2006.

16. Essner R: Experimental frontiers for clinical applications: novel approaches to understanding the mechanisms of lymph node metastases in melanoma. Cancer Metastasis Rev 25: 257-267, 2006. 